\title{
MOdELING CASSIE DROPLETS ON SUPERHYDROPHOBIC COATINGS WITH ORTHOGONAL FIBROUS STRUCTURES
}

\author{
H. Aziz ${ }^{1}$, M.M. Amrei ${ }^{1}$, A. Dotivala ${ }^{2}$, C. Tang $^{2}$, and H. Vahedi Tafreshi ${ }^{1 *}$ \\ ${ }^{1}$ Department of Mechanical and Nuclear Engineering, \\ Virginia Commonwealth University, Richmond, Virginia 23284-3015 \\ ${ }^{2}$ Department of Chemical and Life Sciences, \\ Virginia Commonwealth University, Richmond, Virginia 23284-3015
}

\begin{abstract}
Superhydrophobic coatings comprised of orthogonally layered fibers are studied in this paper in terms of their ability to accommodate water droplets at the non-wetting Cassie state. The effects of microstructural properties of these coatings on droplet contact angles and Cassie state stability are investigated via numerical simulation. More specifically, mathematical expressions are derived to predict whether or not such fibrous coatings can provide sufficient capillary forces for the droplet to remain in the Cassie state. For comparison, similar coatings comprised of parallel fibers are also studied, as a droplet may only interact with the first layer of fibers (parallel fibers) when the fiber spacing is smaller than some critical spacing value. Considerable differences were observed between droplet contact angles on coatings made of orthogonally layered fibers and those having multiple layers of parallel fibers. Our numerical simulations conducted using the Surface Evolver finite element code indicated that apparent contact angle of a droplet can be different in longitudinal and transverse directions, and they both increase by decreasing the diameter of the fibers or by increasing their spacing. It was also found that contact angle in the longitudinal direction is more sensitive to the spacing or the diameter of the fibers. It was also found that a droplet may achieve higher contact angles on a coating with orthogonally layered fibers than on its parallel-fiber counterpart.
\end{abstract}

KEYWORDS: Superhydrophobic surfaces; fibrous coatings; wetting

\footnotetext{
*Address correspondence to Hooman V. Tafreshi, email: htafreshi@ vcu.edu; tel.: 804-828-9936; fax: 804-827-7030; URL: http://www.people.vcu.edu/ htafreshi/
} 


\section{INTRODUCTION}

Superhydrophobic surfaces are generally known for their ability to provide droplet contact angles (CAs) in the neighborhood of 150 degrees, and they are often produced by imparting roughness to a hydrophobic surface (see e.g., [15]). There are two main stable wetting states for a droplet residing on a rough surface, the Wenzel state and the Cassie state (see e.g., [6-9]). The Wenzel state corresponds to the state where the surface asperities are completely submerged in the droplet, whereas the Cassie state represents the condition where a layer of air is trapped underneath the droplet between the peaks of the surface protrusions [10-13]. There are also some other wetting states in between or related to these two extreme states (e.g., as impregnated Cassie state or rose petal state [9]).

While numerous studies have been focused on lowering the cost of micro-fabrication, manufacturing micro- or nanoroughness has remained a costly process. Moreover, adding microfabricated roughness to a surface with arbitrary or random curvatures is still a challenge. An alternative approach (amongst many other methods) has therefore been to impart comparable superhydrophobic behavior to a surface by coating the surface with fibers from a hydrophobic polymer (see e.g., [14-18]). Fibrous coatings usually consist of layers of planar fibers deposited on top of one another in a random fashion. Coatings with random fiber orientations however, do not provide directionality to the mobility of a droplet over the surface. It is expected that controlling the orientation of the fibers in a coating can potentially provide some degrees of control over a droplet mobility on a surface. In fact, it has been shown that droplet can maintain different apparent CAs in different directions on a surface made of parallel grooves for instance, indicating preferential droplet mobility along the grooves (see e.g., [19-28]). While producing a fibrous coating made of parallel fibers that can resemble a grooved surface is not a challenge, controlling the porosity and uniformity of such coatings is quite hard as the fibers tend to pack relatively densely in a thin layer. A possible solution that helps with increasing the spacing between the fibers is to alternate the orientation of the fibers between the $\mathrm{x}$ - and $\mathrm{y}$-directions during the spinning process. Coatings with orthogonally layered fibers tend to have a much higher porosity than their unidirectional counterparts and have been shown to exhibit unique properties for various applications [29-35]. Unfortunately, coatings with orthogonal fibers may not necessarily perform like an anisotropic surface depending on the size and surface tension of the droplets, diameter and 
spacing of the fibers, and the Young-Laplace contact angle (YLCA) of the fiber polymer, as will be discussed later in this paper.

Figure 1a shows a two-layer Polystyrene coating comprised of orthogonally oriented fibers with an average diameter of about $5 \mu \mathrm{m}$. As can be seen in the inset figure, such coatings tend to exhibit large CAs in the neighborhood of 150 degrees (droplet volume is $0.5 \mu \mathrm{l}$ ). Figure $1 \mathrm{~b}$ shows water droplet $\mathrm{CA}$ on different Polystyrene coatings with having the same average fiber diameter but different average fibers spacing. Note that as fiber spacing varies significantly across each coating, we used solid area fraction (SAF) to characterize the density of the fibers in each coating (see [36] for more information on obtaining SAF values from SEM images) as the best alternative, although we recognize that fiber spacing and SAF are only weakly correlated. The experimental measurements given in this figure indicate that apparent contact angle generally decreases with increasing SAF but the trend is not monotonic due to variety of reasons, some of which will be discussed later in this paper. In this paper, we study how fiber diameter and fiber spacing can cause a droplet on a coating with orthogonal fibers departs from the Cassie state, and provide analytical expression for such transition whenever possible. Such information can be quite useful in design superhydrophobic fibrous surfaces for variety of applications such as self-cleaning and anti-icing.

The remainder of this paper is organized as follows. Section 2 presents our force balance method used to predict the stability of the air-water interface (AWI) underneath a droplet deposited on a coating. Section 3 describes our numerical simulation of droplet shape on fibrous coatings surface using the Surface Evolver (SE) finite element code [37]. Results of our simulations and analytical predictions are given in Section 4 followed by our conclusions in Section 5.

\section{ANALYTICAL EQUATIONS FOR AIR-WATER INTERFACE}

As mentioned earlier, a droplet deposited on a fibrous coating may be in the Cassie or Wenzel state depending on the volume of the droplet as well as the coating's microstructural parameters. In this paper, we derive a set of analytical expressions that can be used to predict the condition where a droplet may departure from the Cassie state. 


\subsection{BALANCE OF FORCES ON AN AIR-WATER INTERFACE}

We start by considering the maximum pressure that an AWI between the fibers of a coating underneath a droplet can tolerate before it fail and wet the coating (partially or completely). We first consider a droplet deposited on a coating made of parallel fibers (see Fig. 2). The AWI between two adjacent parallel fibers is shown schematically in Fig. 3a. To make the expressions more general, the fibers are assumed to have different diameters and YLCAs, and are placed in different horizontal planes. The fiber diameter and YLCA are assumed to be $d_{1}^{f}$ and $\theta_{1}^{Y L}$ for the top layer, and $d_{2}^{f}$ and $\theta_{2}^{Y L}$ for the bottom layer, respectively. Fiber spacing is assumed to be the same for both layers for the sake of simplicity. In a plane cutting through the center of the droplet, the AWI can be represented as an arc of a circle (a 2-D approximation). The forces acting on the AWI are the droplet pressure and the capillary forces from the fibers. Writing the balance of these two forces in the direction of $z^{\prime}$ axis, which passes through the middle of the line AB (see Fig. 3a), one can relate the pressure acting on the AWI $P_{F B}^{\square}$ to the location of the AWI between the fibers,

$$
P_{F B}^{\square}=-2 \sigma \cos \phi\left\{\frac{\sin \left(\theta_{1}^{Y L}+\alpha_{1}-\phi\right)+\sin \left(\theta_{2}^{Y L}+\alpha_{2}+\phi\right)}{s-d_{1}^{f} \sin \alpha_{1}-d_{2}^{f} \sin \alpha_{2}}\right\}
$$

where, $\phi=\tan ^{-1}\left\{\frac{2 c+d_{1}^{f} \cos \alpha_{1}-d_{2}^{f} \cos \alpha_{2}}{s-d_{1}^{f} \sin \alpha_{1}-d_{2}^{f} \sin \alpha_{2}}\right\}$ is the angle between the $z^{\prime}$ axis and the vertical direction, and $c$ is the vertical center-to-center distance between the fibers in the first and second layers. In this equation, $\alpha_{1}$ and $\alpha_{2}$ are the immersion angles. The maximum pressure that an AWI between two parallel fibers can withstand can be calculated by solving the following coupled equations for $\alpha_{1}$ and $\alpha_{2}$, and then by substituting these values in Eq. 1 .

$$
\frac{\partial P_{F B}^{\square}}{\partial \alpha_{1}}=0 \quad \& \frac{\partial P_{F B}^{\square}}{\partial \alpha_{2}}=0
$$

Figures $3 \mathrm{~b}$ and $3 \mathrm{c}$ show a schematic drawing of the AWI in a coating with orthogonal fiber-layers, from two different directions of along and perpendicular to the fibers in the top layer. Once again, one can relate the pressure over the AWI $P_{F B}^{\perp}$ to the location of the AWI between the fibers by writing the balance of force in the vertical direction. In our derivations, the changes in $\alpha_{1}$ and $\alpha_{2}$ along the length of the fibers were neglected for simplicity. The force in the vertical direction due to droplet pressure can be taken as $P_{F B}^{\perp}\left(s_{1}-d_{1}^{f} \sin \alpha_{1}\right)\left(s_{2}-d_{2}^{f} \sin \alpha_{2}\right)$. This force is balanced by the 
vertical components of fiber capillary forces $2 \sigma\left(s_{2}-d_{2}^{f} \sin \alpha_{2}\right)$ and $2 \sigma\left(s_{1}-d_{1}^{f} \sin \alpha_{1}\right)$. From the balance of forces, we obtain,

$$
P_{F B}^{\perp}=-2 \sigma\left\{\frac{\sin \left(\theta_{1}^{Y L}+\alpha_{1}\right)}{s_{1}-d_{1}^{f} \sin \alpha_{1}}+\frac{\sin \left(\theta_{2}^{Y L}+\alpha_{2}\right)}{s_{2}-d_{2}^{f} \sin \alpha_{2}}\right\}
$$

where $s_{1}$ and $s_{2}$ are the center-to-center distance between the fibers in the first and second layers, respectively. Maximum $P_{F B}^{\perp}$ can again be found by taking the derivative of Eq. 3 with respect to $\alpha_{1}$ and $\alpha_{2}$ and by solving the resulting coupled equation using the same approach presented earlier.

It is important to note that an AWI may come into contact with the substrate underneath the fibers at pressures much smaller than those predicted by Eqs. 1-3 for certain coating geometries (fiber diameters, fiber spacing, or YLCAs). It is also possible that the AWI meets and coalesces with itself inside the coating under the first layer of fibers resulting in partial or complete wetting transition of the coating. These conditions are explained in the next two subsections.

\subsection{CAssie-to-WenZel Transition. Mechanism I: InTERface SAgGing}

The first scenario of a droplet transitioning from the Cassie state is when the droplet comes into contact with the hydrophilic substrate underneath the coating before the pressure over its AWI exceeds the coating's maximum capillary pressure [15]. Consider a droplet deposited on a coating made of parallel fibers. An AWI between two adjacent fibers is shown schematically in Fig. 4a for when the AWI reaches deep into the coating to touch the substrate. It can be shown that the radius of curvature of this AWI $R_{s a g}^{f, \square}$ can be derived as,

$$
R_{s a g}^{f, \square}=\frac{\left[\left\{\frac{m s^{2} \zeta_{2}}{d_{1}^{f}}+\frac{1}{d_{1}^{f}}\left\{\frac{2 c}{d_{1}^{f}}-\left(1-\zeta_{1}\right)+m\left(1-\zeta_{2}\right)\right\}\left\{s^{2}+4 c^{2}+d_{1}^{f 2}\left(m^{2}-1\right)+4 c m d_{1}^{f}\right\}\right\}^{1 / 2}-\left\{\frac{m s^{2} \zeta_{2}}{d_{1}^{f}}\right\}^{1 / 2}\right]^{2}}{4\left\{\frac{2 c}{d_{1}^{f}}-\left(1-\zeta_{1}\right)+m\left(1-\zeta_{2}\right)\right\}^{2}}
$$


where $m=\frac{d_{2}^{f}}{d_{1}^{f}}, \quad \zeta_{i}=1-\cos \theta_{i}^{Y L}$ with subscript $i=1,2$ representing the layers and $c \neq 0$ when $d_{1}^{f}=d_{2}^{f}=d^{f}$ and $\theta_{1}^{Y L}=\theta_{2}^{Y L}=\theta^{Y L}$. The AWI's transition pressure $P_{\text {sag }}^{f, \square}$ can then be obtained from Laplace’s theorem,

$$
P_{s a g}^{f, \square}=\frac{\sigma}{R_{s a g}^{f, \square}}
$$

Equation 4 can significantly be simplified for when the fibers of the top and bottom layers have the same diameters and YLCAs, i.e., $d_{1}^{f}=d_{2}^{f}=d^{f}, \theta_{1}^{Y L}=\theta_{2}^{Y L}=\theta^{Y L}$, and $c=d^{f}$.

$$
R_{s a g}^{f, \square}=\left[\left\{\frac{s^{2}(2+\zeta)}{16 d^{f}}+d^{f}\right\}^{1 / 2}-\left\{\frac{s^{2} \zeta}{16 d^{f}}\right\}^{1 / 2}\right]^{2}
$$

For given values of $s, d^{f}$ and $\theta^{Y L}$, transition pressure $P_{\text {sag }}^{f, \square}$ for an AWI over parallel layers of fibers can be calculated using Eqs. 4-6. For instance, with $d^{f}=10 \mu \mathrm{m}, \theta^{Y L}=85^{\circ}$, and $s=264 \mu \mathrm{m}$, this pressure becomes equal to the Laplace pressure for a droplet with a volume of $0.524 \mu \mathrm{l}$. In other words, a droplet with a volume of $0.524 \mu 1$ will not remain at the Cassie state on such coatings if $s \geq 264 \mu \mathrm{m}$. Figure $4 \mathrm{~b}$ shows the variation of $P_{s a g}^{f, \square}$ with $s$ for a surface coated with parallel fibers with different diameters. It can be seen that $P_{\text {sag }}^{f, \square}$ decreases with increasing fiber spacing, but it increases with increasing the diameter of the fibers in the lower layer.

Figures $5 \mathrm{a}$ and $5 \mathrm{~b}$ show a schematic illustration for the AWI over a coating with orthogonal fibers from two different views of along and perpendicular to the fibers in the upper layer. These figures are drawn for when the AWI comes into contact with the substrate. Assuming that the AWI in a plane passing through the droplet center is part of an ellipsoid, the radius of curvature of the AWI will be the harmonic mean of the radii of curvature of the circular arcs shown in Figs. 5a and 5b. Each layer of fibers has a different fiber diameter, fiber spacing, or YLCA. These radii of curvature can be derived to be, 


$$
\begin{gathered}
R_{s a g, 1}^{f, \perp}=\frac{\frac{s_{1}^{2}}{4 d_{1}^{f}}+m^{2} d_{1}^{f}+m d_{1}^{f}}{2 m+\zeta_{1}} \\
R_{s a g, 2}^{f, \perp}=\frac{s_{2}^{2}}{4 d_{2}^{f} \zeta_{2}}
\end{gathered}
$$

The transition pressure for the AWI over orthogonal layers of fibers can then be obtained as,

$$
P_{s a g}^{f, \perp}=\sigma\left(\frac{1}{R_{s a g, 1}^{f, \perp}}+\frac{1}{R_{s a g, 2}^{f, \perp}}\right)
$$

From Eqs. 7-9, one can obtain,

$$
P_{s a g}^{f, \perp}=4 \sigma d_{1}^{f}\left[\frac{2 m+\zeta_{1}}{s_{1}^{2}+4 d_{1}^{f 2}\left(m^{2}+m\right)}+\frac{m \zeta_{2}}{s_{2}^{2}}\right]
$$

For the special case of $d_{1}^{f}=d_{2}^{f}=d^{f}, s_{1}=s_{2}=s$, and $\theta_{1}^{Y L}=\theta_{2}^{Y L}=\theta^{Y L}$, Eq. 10 simplifies to the following equation.

$$
P_{\text {sag }}^{f, \perp}=4 \sigma d^{f}\left(\frac{2+\zeta}{s^{2}+8 d^{f 2}}+\frac{\zeta}{s^{2}}\right)
$$

For instance, with $d^{f}=10 \mu \mathrm{m}, \theta^{Y L}=85^{\circ}$, and $s=194.1 \mu \mathrm{m}$, this pressure becomes equal to the Laplace pressure for a droplet with a volume of $0.524 \mu 1$, and so such a droplet will not stay at the Cassie state on such coatings if $s \geq 194 \mu \mathrm{m}$. Figure $5 \mathrm{c}$ shows the variation of $P_{\text {sag }}^{f, \perp}$ with fiber spacing for a coating with orthogonal layers of fibers having different diameters. It can be seen that $P_{\text {sag }}^{f, \perp}$ decreases with the increasing $s$, but it increases with the increasing the diameter of the fibers in the bottom layer. Figure $5 \mathrm{~d}$ shows the transition pressures versus fiber spacing for $d^{f}=10 \mu \mathrm{m}, \theta^{Y L}=85^{\circ}$, $\theta^{Y L}=100^{\circ}$ for both coatings with parallel and orthogonal layers of fibers. It can be observed that $P_{s a g}^{f, \square}$ is always higher than $P_{\text {sag }}^{f, \perp}$ when the fiber spacing, fiber diameter, and YLCA are kept constant. This means that a droplet deposited on coating with parallel fibers transitions from the Cassie state at a greater fiber spacing compared to the same droplet sitting on a coating with orthogonal fiber-layers with the same fiber diameter and YLCA. 


\subsection{CASSIE-TO-WenZel TRANSITION. MeChanism II: InTERFACE COALESCENCE}

Another cause of departure from the Cassie state is the coalescence of different segments of the AWI inside a coating in such a way that it results in the submersion of a fiber-layer [26]. As depicted in Fig. 6, if the diameter of the fibers in the bottom layer is large enough, the AWI from the lateral sides of a fiber may come into contact with one another and coalesce into one AWI underneath the fiber. Consider the transition pressure for an AWI over a coating with parallel fibers as shown in Fig. 6a. Each layer of fibers has different fiber diameter and YLCA, but the fiber spacing is the same for both layers. The radius of curvature of the AWI when it is about to meet itself below the first layer should satisfy the following equation.

$$
\left(s+d_{1}^{f} \cos \theta_{1}^{Y L}-d_{2}^{f} \cos \theta_{2}^{Y L}\right) R_{\text {coal }}^{f, \square}+\left(d_{1}^{f}+d_{2}^{f}\right)\left(\frac{d_{1}^{f 2}}{4}-R_{\text {coal }}^{f, \square} d_{1}^{f} \cos \theta_{1}^{Y L}\right)^{1 / 2}=\frac{s^{2}}{4}+\frac{d_{1}^{f}}{2}\left(d_{1}^{f}+d_{2}^{f}\right)
$$

Departure from the Cassie state takes place through this mechanism for coatings if $d_{2}^{f}>h^{\square}$. Here, $h^{\square}$ is the height of the AWI below the first layer at the moment of coalescence (see Fig. 6a), and it can be calculated as,

$$
h^{\square}=\left(\frac{d_{1}^{f 2}}{4}-R_{\text {sag }}^{f, \square} d_{1}^{f} \cos \theta_{1}^{Y L}\right)^{1 / 2}-\frac{d_{1}^{f}}{2}+R_{\text {sag }}^{f, \square}
$$

Transition pressure for such coatings $P_{\text {coal }}^{f, \square}$ can then be calculated using Eq. 12 and the Laplace equation,

$$
P_{\text {coal }}^{f, \square}=\frac{\sigma}{R_{\text {coal }}^{f, \square}}
$$

Figures $6 \mathrm{~b}$ and $6 \mathrm{c}$ show the transition through AWI coalescence in coatings comprised of orthogonal fibers. For such coatings, the transition takes place when $d_{2}^{f}>h^{\perp}$ where $h^{\perp}$ is the height of the AWI when it is about to meet itself below the first layer (see Fig. 6b), i.e.,

$$
h^{\perp}=\frac{d_{1}^{f}}{2}\left(1-\frac{2 s_{1} \cos \theta_{1}^{Y L}}{d_{1}^{f}}\right)^{1 / 2}+\frac{1}{2}\left(s_{1}-d_{1}^{f}\right)
$$

The AWI radii of curvature can be calculated to be,

$$
R_{\text {coal }, 1}^{f, \perp}=\frac{S_{1}}{2}
$$




$$
R_{\text {coal }, 2}^{f, \perp}=\frac{s_{2}^{2}+4 h^{\perp}\left(h^{\perp}-d_{2}^{f}\right)}{4\left(2 h^{\perp}-d_{2}^{f}-d_{2}^{f} \cos \theta_{2}^{Y L}\right)}
$$

The transition pressure $P_{c o a l}^{f, \perp}$ can again be obtained using the Laplace equation,

$$
P_{\text {coal }}^{f, \perp}=2 \sigma\left[\frac{1}{s_{1}}+\frac{2\left(2 h^{\perp}-d_{2}^{f}-d_{2}^{f} \cos \theta_{2}^{Y L}\right)}{s_{2}^{2}+4 h^{\perp}\left(h^{\perp}-d_{2}^{f}\right)}\right]
$$

Figure 7a shows transition pressure for coatings with parallel and orthogonal fibers versus fiber spacing in the bottom layer $s_{2}$. It is important to note that $s_{1}$ is equal to $s_{2}$ for coatings with parallel fibers, but this is not the case for coatings made of orthogonal layers. For both coatings $d_{1}^{f}=1 \mu \mathrm{m}, d_{2}^{f}=20 \mu \mathrm{m}$, and $\theta_{1}^{Y L}=\theta_{2}^{Y L}=100^{\circ}$, and $s_{1}=30 \mu \mathrm{m}$. It can be seen that $P_{\text {coal }}^{f, \perp}=P_{\text {sag }}^{f, \perp}$ at the $s_{2}$ value for which $h^{\perp}=d_{2}^{f}$. For smaller fiber spacing, departure from the Cassie state occurs due to AWI coalescence, and the transition pressure can be calculated using Eq. 18. Similarly, $P_{\text {coal }}^{f, \square}=P_{\text {sag }}^{f, \square}$ at the $s_{2}$ value for which $h^{\square}=d_{2}^{f}$. For smaller spacing, the transition happens due to AWI coalescence, and $P_{\text {coal }}^{f, \square}$ can be calculated using Eqs. 12 and 14. Figure 7a also shows that for all $s_{2}$ values, $P_{F B, \max }^{\square}$ is higher than $P_{s a g}^{f, \square}$ and $P_{\text {coal }}^{f, \square}$, while $P_{F B \text {,max }}^{\perp}$ remains higher than $P_{\text {sag }}^{f, \perp}$ and $P_{\text {coal }}^{f, \perp}$. Therefore, for the conditions considered in this paper, transition from the Cassie state takes place either due to the AWI coming into contact with the substrate or coalescing with itself below the first layer. Figure 7b shows an example of a droplet with a volume of $0.524 \mathrm{~mm}^{3}$ deposited on a two-layer coating composed of parallel fibers ( $d^{f}=10 \mu \mathrm{m}, \theta^{Y L}=100^{\circ}$, and $\left.s=170 \mu \mathrm{m}\right)$. Figure $7 \mathrm{c}$ shows the same droplet on a similar coating but with orthogonal fibers. Two additional magnified images are added to these figures to better show the AWI between consecutive fibers in a plane cutting through the center of the droplet. The magnified images clearly show that a transition due to sagging mechanism is about to happen upon further increasing the fiber spacing in both cases.

The equations derived in this paper can be used to predict if a droplet can achieve the Cassie state on a coating with parallel or orthogonal fibers. For instance, consider a droplet with a volume of $0.524 \mathrm{~mm}^{3}$ (a Laplace pressure of $288 \mathrm{~Pa}$ assuming a spherical droplet) on a coating with parallel layers of fibers with $d^{f}=10 \mu \mathrm{m}$ and $\theta^{Y L}=100^{\circ}$. To determine 
the fiber spacing at which departure from the Cassie state takes place, one can assume a transition pressure of $P_{s a g}^{f, \square}=288$ Pa in Eq. 5 and then solve this Eqs. 5 and 6 for $s$ (resulting in $s=284.2 \mu \mathrm{m}$ ). Similar calculations can be conducted for the orthogonal counterpart of this coating using in Eq. 16 to obtain a fiber spacing of $s=207.1 \mu \mathrm{m}$. Likewise, one can calculate a critical fiber spacing a given droplet on coatings with different microstructural parameters. Figure 8 shows the critical fiber spacing versus fiber diameter for coatings with parallel and orthogonal fibers at a Laplace pressure of $288 \mathrm{~Pa}$ and a YLCA of $\theta^{Y L}=100^{\circ}$. This figure compares the predictions of our analytic calculations with those of SE simulations. It was found that $s^{c}$ is greater for coatings with parallel fibers compared to their orthogonal counterparts. This is because of the staggered arrangement of fibers in coatings with parallel fibers, where the AWI is supported by a fiber from the top layer and a fiber from the bottom layer (see Fig. 7b).

\section{Modeling Droplet Shape on Fibrous CoATINGS}

The roughness geometry of a coating comprised of parallel fibers is different from that of a coating made up of orthogonal of fibers. As such, one expects to observe different apparent CAs on these coatings. The CAs are expected to depend on fiber diameter, fiber spacing, and YLCA of the fiber material which will be discussed later in this paper. Among different methods reported in the literature [38-41] for modeling droplet shape on a surface, Surface Evolver (SE) code was considered in our study for its modest computational requirements and its availability in the public domain [37]. SE computes the equilibrium shape of a droplet by minimizing the free energy of the fiber-droplet system [37]. The system's free surface energy can be expressed as:

$$
E=\sigma A_{a w}-\sigma \cos \theta^{Y L} \iint_{A_{s w}} d A
$$

The subscripts $a, w$, and $s$ denote surrounding air, water, and solid (fibers), respectively. In this equation, $A_{a w}$ and $A_{s w}$ are surface areas of the AWI and solid-water interface. The effects of gravity are not included in our work, as the droplets are assumed to be fairly small. To calculate fibers' energy contribution, the integrand $d A$ in Eq. 19 must be derived for each 
fiber in the simulation domain. For the sake of simplicity, the fibers of the top layer are aligned in the y-direction for all the coatings discussed in this work, i.e.,

$$
d A=-\frac{y z}{\sqrt{x^{2}+z^{2}}} d x+\frac{x y}{\sqrt{x^{2}+z^{2}}} d z
$$

For a energy calculation, the volume of the fibers must be removed from the total volume under the AWI. This is done here by deriving an expression for the fiber's volume element and incorporating that in the SE calculations, i.e.,

$$
d V=y z d x
$$

For coatings with orthogonal layers, the area and volume elements for the fibers in the bottom layer (aligned in the $x$ direction) should also be described mathematically and programed in SE. With the customized area and volume elements, SE can produce the equilibrium shape of a droplet by minimizing the energy of the system while keeping the droplet volume constant. Starting from an arbitrary initial shape (represented with a piecewise linear set of triangular facets), SE can obtain the equilibrium shape of a droplet iteratively. Longitudinal and transverse apparent contact angles were measured from the full size longitudinal and transverse images of the droplet on a computer screen. In the absence of a universally accepted contact angle measurement method for a droplet on a rough surface, we estimated droplet apparent contact angles, at the intersection point of a horizontal baseline (drawn $6-8 \mu \mathrm{m}$ above the coating's top surface) and the droplet profile. Obviously, close attention was paid to the consistency and reproducibility of these readings.

\section{RESUlTS AND DiSCUSSION}

\subsection{EFFeCts of Fiber Diameter on APPARENT Contact ANGLE}

Consider a droplet with a fixed volume deposited on a coating comprised of parallel fibers. Since the apparent CA is not uniform along the droplet contact line, it is expected that the apparent CA observed from a direction parallel to the fibers (longitudinal) will be different from that from a direction perpendicular to the fibers (transverse) as shown in Fig. 9a-9c.

Figure 9d shows how longitudinal and transverse apparent CAs vary with fiber diameter for coatings with parallel fibers having a fiber spacing of $s=40 \mu \mathrm{m}$. As expected, apparent CAs are greater than the YLCA in all cases, as the contact area between the droplet and solid surface is reduced in the Cassie state. More importantly, it can be seen that apparent 
CAs decrease with increasing fiber diameter. This is because the number of fibers on which the droplet sits increases with increasing the fiber diameter for a given fiber spacing. Therefore, the total wetted area of the coating increases with increasing fiber diameter. It can also be seen in Fig. 9d that longitudinal apparent CA is greater than its transverse counterpart (see Figs. 9a-9c). Figure 9e presents the wetted area of the coating under the droplet. It can be seen that wetted area increases with increasing fiber diameter and it is always higher for coatings with smaller YLCA. The inset in Fig. 9e shows the fibers wetted length in planes cutting through the middle of the droplet in longitudinal and transvers directions. It can be seen that wetted length in the transverse direction is larger than that in the longitudinal direction especially when the fibers are thin. This seems to correlate well with how apparent CAs in the longitudinal and transvers directions behave in Fig. 9d, where a longer wetted length is observed for smaller apparent CAs.

Although we started this subsection by assuming a coating comprised of parallel fibers, it is important to note that the above discussion also applies to coatings with orthogonal fibers as long as the spacing between the fibers is small enough for the droplet to remain in contact with the first layer only (the case shown in Fig. 9). It is in fact quite easy to analytically calculate the maximum fiber spacing below which a given droplet never comes into contact with the fibers in the second layer, regardless of the orientation of the fibers in the second layer. One can calculate the pressure at which the AWI deflects as much as one fiber diameter to reach the fibers in the second layer (assuming the point of contact with the fibers in the second layer is at an equal distance from the centers of the fibers in the first layer, i.e., the AWI is symmetric).

Let us consider a droplet deposited on the first layer of fibers in a fibrous coating. Using the Cassie-Baxter equation, for the apparent $\mathrm{CA}$ in the longitudinal direction (the Cassie-Baxter equation does not apply to transverse CAs), one can obtain,

$$
\cos \theta_{A}^{L}=\frac{\alpha d^{f}}{s} \cos \theta^{Y L}+\frac{d^{f} \sin \alpha}{s}-1
$$

where $\alpha$ is the immersion angle, and it is dependent on droplet pressure, fiber diameter, fiber spacing, and YLCA [26,42]. 


$$
P_{d r o p}=-\frac{2 \sigma \sin \left(\theta^{Y L}+\alpha\right)}{\left(s-d^{f} \sin \alpha\right)}
$$

Predictions of Eqs. 22 and 23 are added to Fig. 9d, and show good agreement with the results of our numerical simulations. Note that the effects of fiber diameter on droplet apparent CA for when the droplet is in contact with both fiber-layers (i.e., for large fiber spacing values) is not discussed in this subsection, for the sake of brevity.

It is interesting to note that the longitudinal CA value of $\theta_{A}^{L}=144^{\circ}$ shown in Fig. $9 \mathrm{~d}$ for a coating with a fiber diameter of $5 \mu \mathrm{m}$ is within $5 \%$ of its experimental counterpart given in Fig. $1 \mathrm{~b}$ (i.e., $\theta_{A}^{L}=152^{\circ}$ ) for a SAF 0.23 which corresponds to the SAF the virtual orthogonal coating used in Fig. 9d.

\subsection{EFFECTS OF FibER SPACING ON APPARENT CONTACT ANGLE}

The effects of fiber spacing on $\theta_{A}^{L}$ and $\theta_{A}^{T}$ in coatings with an arbitrary fiber diameter of $10 \mu \mathrm{m}$ is studied in this subsection. As mentioned earlier in Sec. 3.1, increasing the fiber spacing can result in the droplet coming into contact with the fibers in the bottom layer. The wetting behavior of a droplet becomes quite different when it is in contact with more than one layer of fibers, and that behavior is different depending on whether the second layer is perpendicular or parallel to the fibers in the top layer (see Figs. 10a-10i). For the set of parameters considered in the present study, the critical fiber spacing is found to be $s_{t}=95.54 \mu \mathrm{m}$ for coatings with an YLCA of $\theta^{Y L}=85^{\circ}$ and, $s_{t}=108.3 \mu \mathrm{m}$ for those with a YLCA of $\theta^{Y L}=100^{\circ}$. It can be seen in Figs. $10 \mathrm{j}-10 \mathrm{k}$ that both the longitudinal and transverse CAs increase almost monotonically with $s$ for $s<s_{t}$ (the number of fibers on which the droplet sits decreases and so the coating's wetted area decreases). Predictions of Eq. 22 (applicable to longitudinal direction only) are also added to Figs. 10j and 10k for $s<s_{t}$, and good general agreement with the results of our simulations can be observed. For $s \geq s_{t}$, the droplet sits on two layers of fibers, and the variation in the longitudinal and transverse CAs with $s$ depends strongly on the orientation of the fibers in the second layer (see Figs. 10a-10i). The longitudinal CA seems to periodically decrease and increase in different ranges of fiber spacing (e.g., from about 100 to $200 \mu \mathrm{m}$ for the case of coatings with parallel fibers and an YLCA of $\theta^{Y L}=85^{\circ}$ ) for 
both the orthogonal and parallel-fiber coatings. This behavior can be explained by considering the number of fibers in contact with the droplet for each fiber spacing. Note that immersion angle increases with increasing the spacing between the fibers, when the number of fibers in contact with the droplet is fixed. This in turn increases the coating's wetted line in the longitudinal direction, and consequently decreases the longitudinal CA. Further increase in the fiber spacing forces the droplet to detach from some of the fibers farther away from the center. This decreases the coating's wetted line in the longitudinal direction and increases the longitudinal CA. Further increase in the fiber spacing repeats the above effects, leading to the reported fluctuating longitudinal CAs. Note that despite these fluctuations, the net effect of increasing the fiber spacing is an overall (yet marginal) increase in the longitudinal CA. Similar variations in apparent CA was has also been reported for droplets deposited on a chemically heterogeneous substrate $[19,43]$. The data given in Figs. 10j and 10k seem to indicate that CA fluctuations become less significant when droplet diameter (about $1 \mathrm{~mm}$ here) is more one order of magnitude larger than 1 fiber spacing.

Transverse CA, unlike its longitudinal counterpart, seems to be relatively independent of fiber spacing (transverse wetted length varies insignificantly with fiber spacing as shown in Figs. 10a-10i). It is interesting to note a sudden drop in transvers CAs given in Figs. 10j and 10k for coatings with parallel fibers. This is accompanied with a sudden increase in the wetted length of the coatings in the transvers direction at the moment a droplet penetrates deep into the coating to reach the second layer of fibers (not shown with numbers for the sake of brevity but can be seen in Figs. 10c-10i).

Overall, it can be seen that longitudinal CAs are greater than their transverse counterparts for both the orthogonal and parallel-fiber coatings. We observed for $s \geq s_{t}$ that, CA on a coating with orthogonal fibers is higher than that of a similar coating with parallel fibers. However, a droplet deposited on the orthogonal coating is more susceptible to fiber spacing, and it may depart from the Cassie state earlier (as the droplet evaporates, for instance) than the same droplet on the coating with parallel fibers. Our analytical equations for a droplet with a volume $0.524 \mu 1$ (see Secs. 2.2 and 2.3) indicated a departure from the Cassie state at a spacing of $s=194.1 \mu \mathrm{m}$, for $\theta^{Y L}=85^{\circ}$, and $s=207.1 \mu \mathrm{m}$, for $\theta^{Y L}=100^{\circ}$, on a coating with orthogonal fibers, and at $s=264 \mu \mathrm{m}$, for $\theta^{Y L}=85^{\circ}$, and $s=284.2 \mu \mathrm{m}$, for $\theta^{Y L}=100^{\circ}$ on the same coating but with parallel fibers. 
Maintaining the Cassie state on a fibrous coating depends on droplet volume, fiber diameter, fiber spacing, and YLCA of the fiber material. One can define the smallest YLCA for which an apparent contact angle greater than $90^{\circ}$ is attainable as the minimum YLCA $\theta_{90}^{Y L \min }$ for a fibrous coating to be hydrophobic. To do so, one needs to first find the minimum YLCAs required for a droplet to remain at the Cassie state $\theta_{C a s}^{Y L \min }$, and then among them, find those that correspond to an apparent contact angle greater than $90^{\circ}$ (i.e., $\theta_{90}^{Y L \min }$ ). Let us consider the simple case of a droplet on a coating with a single layer of parallel fibers. It can be shown that, departure from the Cassie state (due to interface sagging) happens only when the following equation is satisfied,

$$
\cos \theta_{C a s}^{Y L \min }=1-\frac{P_{d r o p} s^{2}}{4 \sigma d^{f}}
$$

It can clearly be seen from Eq. 24, that $\cos \theta_{\text {Cas }}^{Y L \min }$ is proportional to $s^{2}$ but inversely proportional to $d^{f}$ (i.e., stronger dependence on fiber spacing). In using this equation, note that $s>d^{f}$. This is in conceptual agreement with the work of [12] and [44] who showed surfaces with re-entrant geometry may exhibit hydrophobic behavior even with YLCAs less than 90 degrees. Note however that, if the sparing between the fibers becomes so small that the fibers come into contact with one another, the coating geometry will no longer provide a re-entrant structure, and no Cassie droplet should be expected for $\theta^{Y L}<90^{\circ}$.

Equations 22 and 23 can be used to calculate $\theta_{90}^{Y L \min }$ by assuming $\theta_{A}^{L}=90^{\circ}$, i.e.,

$$
\cos \theta_{90}^{Y L \min }=\frac{s}{\alpha d^{f}}-\frac{\sin \alpha}{\alpha}
$$

and

$$
P_{d r o p}=-\frac{2 \sigma \sin \left(\theta_{90}^{Y L \min }+\alpha\right)}{\left(s-d^{f} \sin \alpha\right)}
$$


For instance, for a droplet with a volume of $0.524 \mu \mathrm{l}$ on a coating comprised of a single layer of fibers with $d^{f}=5 \mu \mathrm{m}$ and $s=5.5 \mu \mathrm{m}$, one obtains $\theta_{\text {Cas }}^{Y L \min }=6.31^{\circ}$ and $\theta_{90}^{Y L \min }=86.4^{\circ}$. This means that such a coating can exhibit hydrophobic behavior for $\theta^{Y L}>86.4^{\circ}$. To confirm that the droplet is at the Cassie state, one should check if $\theta_{90}^{Y L \min }>\theta_{\text {Cas }}^{Y L \min }$.

\section{CONCLUSIONS}

The Cassie state of a droplet deposited on a coating with orthogonal fibers is studied in this paper. For comparison, similar coatings comprised of parallel fibers are also studied, as a droplet may only interact with a layer of parallel fibers when the fiber spacing is smaller than some critical spacing value. Easy-to-use analytical expressions are derived to predict the condition in which a droplet may depart from the non-wetting Cassie state by partially or completely wetting the coating below it. The numerical simulations conducted in this work indicate that apparent contact angles of a droplet can be increased by decreasing the diameter of the fibers in the coating for a given fiber spacing (fiber count per unit length). Similarly, it was shown that increasing the fiber spacing, up to a critical value, can also help to increase the contact angles on a coating with a given fiber diameter. However, droplet contact angle can exhibit considerable fluctuations with varying fiber spacing. Considerable differences was observed between droplet contact angles on orthogonally layered and parallel-fiber coatings, i.e., a droplet may achieve higher contact angles on a coating with orthogonal fibers.

\section{ACKNOWLEDGEMENT}

HVT and MMA acknowledge National Science Foundation CMMI (Grant No. 1029924) and CBET (Grant No. 1402655) programs for partial support of this research. 


\section{NOMENCLATURE}

$A=$ area

$A_{a w}=$ area of the air-water interface

$A_{s w}=$ area of the solid-water interface

$c=$ vertical center-to-center distance between fibers in the first and second layers in coatings with parallel fibers

$d^{f}=$ fiber diameter

$E=$ energy

$h^{\square}=$ height of the AWI at the moment of AWI coalescence in coatings with parallel fibers

$h^{\perp}=$ height of the AWI when it is about to coalesce with itself in coatings with orthogonal fibers

$m=$ ratio of the diameter of the fibers in the bottom layer to that in the top layer

$P_{d r o p}=$ Laplace pressure for a droplet

$P_{\text {coal }}^{f, \square}=$ transition pressure due to AWI coalescence, coatings with parallel fibers

$P_{\text {coal }}^{f, \perp}=$ transition pressure due to AWI coalescence, coatings with orthogonal fibers

$P_{F B}^{\square}=$ capillary pressure from force balance, coatings with parallel fibers

$P_{F B}^{\perp}=$ capillary pressure from force balance, coatings with orthogonal fibers

$P_{s a g}^{f, \square}=$ transition pressure due to AWI sagging, coatings with parallel fibers

$P_{\text {sag }}^{f, \perp}=$ transition pressure due to AWI sagging, coatings with orthogonal fibers

$R_{\text {coal }}^{f, \square}=$ AWI radius of curvature at the moment of coalescence, coatings with parallel fibers

$R_{c o a l, i}^{f, \square}=\mathrm{AWI}$ radii of curvature at the moment of coalescence, coatings with orthogonal fibers $(i=1,2)$

$R_{s a g}^{f, \square}=$ AWI radius of curvature when it sags to reach the substrate, coatings with parallel fibers

$R_{s a g, i}^{f, \perp}=$ AWI radii of curvature when it sags to reach the substrate, in coatings with orthogonal fibers $(i=1,2)$

$s=$ center-to-center spacing between two fibers in the same layer

$s^{c}=$ fiber spacing for which the AWI reaches the substrate

$s_{t}=$ fiber spacing for which the AWI reaches the second layer of fibers

$V^{d}=$ volume of droplet 
$\alpha=$ immersion angle

$\theta^{Y L}=$ Young-Laplace contact angle

$\theta_{A}^{L}=$ apparent contact angle observed from the direction parallel to the fibers in the top layer

$\theta_{A}^{T}=$ apparent contact angle observed from the direction perpendicular to the fibers in the top layer

$\theta_{\text {Cas }}^{Y L \min }=$ Minimum YLCA required for a droplet to remain at the Cassie state

$\theta_{90}^{Y L \min }=$ Minimum YLCA required for a droplet to have an apparent contact angle greater than $90^{\circ}$

$\sigma=$ surface tension 


\section{REFERENCES}

[1] D. Quere, Wetting and roughness, Annu. Rev. Mater. Res. 38 (2008) 71-99.

[2] N.J. Shirtcliffe, G. McHale, S. Atherton, M.I. Newton, An introduction to superhydrophobicity, Adv. Colloid Interf. Sci. 161 (2010) 124-138.

[3] J.P. Rothstein, Slip on superhydrophobic surfaces, Annu. Rev. Fluid Mech. 42 (2010) 89-109.

[4] C. Lee, C.-H. Choi, C.-J. Kim, Structured surfaces for giant liquid slip, Phys. Rev. Lett. 101 (2008) 064501.

[5] C.W. Extrand, Repellency of the lotus leaf: resistance to water intrusion underhydrostatic pressure, Langmuir 27 (2011) 6920-6925.

[6] J.D. Eick, R.J. Good, A.W. Neumann, Thermodynamics of contact angles. 2. Rough solid-surfaces, J. Colloid Interface Sci. 53 (1975) 235-248.

[7] B. He, N.A. Patankar, J. Lee, Multiple equilibrium droplet shapes and design criterion for rough hydrophobic surfaces, Langmuir 19 (2003) 4999-5003.

[8] J. Long, M.N. Hyder, R.Y.M. Huang, P. Chen, Thermodynamic modeling of contact angles on rough, heterogeneous surfaces, Adv. Colloid Interface Sci. 118 (2005) 173-190.

[9] E. Bormashenko, Progress in understanding wetting transitions on rough surfaces, Advances in colloid and interface science 222 (2015) 92-103.

[10] R.N. Wenzel, Resistance of solid surfaces to wetting by water, Ind. Eng. Chem. 28 (1936) 988-994.

[11] A.B.D. Cassie, S. Baxter, Wettability of porous surfaces, Trans. Faraday Soc. 40 (1944) 0546-0550.

[12] A. Marmur, Wetting on the hydrophobic rough surfaces: To be heterogeneous or not to be?, Langmuir 19 (2003) $8343-8348$.

[13] E. Bormashenko, Y. Bormashenko, T. Stein, G. Whyman, E. Bormashenko, Why do pigeon feathers repel water? Hydrophobicity of pennae, Cassie-Baxter wetting hypothesis and Cassie-Wenzel capillarity-induced wetting transition, J. Colloid Interface Sci. 311 (2007) 212-216.

[14] M. L. Ma, R. M. Hill, G. C. Rutledge, A review of recent results on superhydrophobic materials based on micro- and nanofibers, J. Adhes. Sci. Technol. 22 (2008) 1799.

[15] A. Tuteja, W. Choi, J. M. Mabry, G. H. McKinely, R. E. Cohen, Robust omniphobic surfaces, Proc. Natl. Acad. Sci. U.S.A. 105 (2008) 18200.

[16] H. Wu, R. Zhang, Y. Sun, D. Lin, Z. Sun, W. Pan, P. Downs, Biomimetic nanofiber patterns with controlled wettability, Soft Matter 4 (2008) 2429-2433.

[17] J. Lin, Y. Cai, X. Wang, B. Ding, J. Yu, M. Wang, Fabrication of biomimetic superhydrophobic surfaces inspired by lotus leaf and silver ragwort leaf, Nanoscale 3 (2011) 1258.

[18] H.S Lim, S.H. Park, S.H. Koo, Y.-J. Kwark, E.L. Thomas, Y. Jeong, J.H. Cho, Superamphiphilic Janus Fabric, Langmuir 26(24) (2010) 19159-19162. 
[19] S. Brandon, A. Wachs, A. Marmur, Simulated contact angle hysteresis of a three-dimensional drop on a chemically heterogeneous surface: a numerical example, J. Colloid Interface Sci. 191 (1997) 110-116.

[20] S. Brandon, N. Haimovich, E. Yeger, A. Marmur, Partial wetting of chemically patterned surfaces: The effect of drop size, J. Colloid Interface Sci. 263 (2003) 237-243.

[21] Y. Chen, B. He, J. Lee, N.A. Patankar, Anisotropy in the wetting of rough surfaces, J. Colloid interface sci. 281 (2004) 458-464.

[22] W. Li, G.P. Fang, Y.F. Lij, G.J. Qiao, Anisotropic wetting behavior arising from superhydrophobic surfaces: parallel grooved structure, J. Phys. Chem. B 112 (2008) 7234-7243.

[23] A.L. Dubov, A. Mourran, M. Moller, O.I. Vinogradova, Contact angle hysteresis on superhydrophobic stripes, Journal of Chemical Physics 141 (2014) 074710.

[24] H.P. Jansen, K. Sotthewes, H.J.W. Zandvliet, E.S. Kooij, Potential of lattice Boltzmann to model droplets on chemically stripe-patterned substrates, Applied Surface Science 361 (2016) 122-132.

[25] L Tie, Z. Guo, W. Liu, Anisotropic wetting properties on various shape of parallel grooved microstructure, J. Colloid Interface Sci. 453 (2015) 142-150.

[26] E. Bormashenko, O. Gendelman, G. Whyman, Superhydrophobicity of lotus leaves versus birds' wings: Different physical mechanisms leading to similar phenomena, Langmuir 28 (2012) 14992 - 14997.

[27] H. Zhao, K.W. Law, Directional self-cleaning of superoleophobic surface, Langmuir 28 (2012) 11812-11818.

[28] S.Z. Wu, J.N. Wang, G.L. Niu, J. Yao, D. Wu, A.W. Li, Reversible switching between isotropic and anisotropic wetting by one-direction curvature tuning on flexible superhydrophobic surfaces, Applied Physics Letters 98 (2011) 081902.

[29] E. Zussman, A. Theron, A.L. Yarin, Formation of nanofiber crossbars in electrospinning, Appl. Phys. Lett. 82 (2003) 6.

[30] T.D. Brown, P.D. Dalton, D.W. Hutmacher, Direct Writing By Way of Melt Electrospinning, Adv. Mater. 23 (2011) $5651-5657$.

[31] B. Emami, H.V. Tafreshi, M. Gad-el-Hak, G.C. Tepper, Effect of fiber orientation on shape and stability of air-water interface on submerged superhydrophobic electrospun thin coatings, Journal of Applied Physics 111 (2012) 064325.

[32] T.M. Bucher, B. Emami, H.V. Tafreshi, M. Gad-el-Hak, G.C. Tepper, Modeling resistance of nanofibrous superhydrophobic coatings to hydrostatic pressures: The role of microstructure, Physics of Fluids 24 (2012) 022109.

[33] P.D. Dalton, C. Vaquette, B.L. Farrugia, T.R. Dargaville, T.D. Brown, D.W. Hutmacher, Electrospinning and additive manufacturing: converging technologies, Biomater. Sci. 1 (2013) 171-185.

[34] T.M. Bucher, H.V. Tafreshi, G.C. Tepper, Modeling Filtraion Performance of Nanofiber Coatings with Orthogonal Fiber Orientations, Powder Technology 249 (2013) 43-53.

[35] T.M. Bucher, M.M. Amrei, H.V. Tafreshi, Wetting resistance of heterogeneous superhydrophobic coatings with orthogonally layered fibers, Surface \& Coatings Technology 277 (2015) 117-127. 
[36] M.A. Samaha, H.V. Tafreshi, M. Gad-el-Hak, Effects of Hydrostatic Pressures on Drag-Reduction Performance of Submerged Aerogel Particle Coatings, Colloids and Surfaces A: Physicochem. Eng. Aspects 399 (2012) 62-70.

[37] K.A. Brakke, Surface Evolver Manual Version 2.50, 2012.

[38] Y. H. Kim, W. Choi, J. S. Lee, Water droplet properties on periodically structured superhydrophobic surfaces: A lattice Boltzmann approach to multiphase flows with higher water/air density ratio, Microfluid Nanofluid 10 (2011) 173 185.

[39] A. Dupuis, J. M. Yeomans, Modeling droplets on superhydrophobic surfaces: Equilibrium states and transitions, Langmuir 21 (2005) $2624-2629$.

[40] A.L. Dubov, A. Mourran, M. Moller, O.I. Vinogradova, Regimes of wetting transitions on superhydrophobic textures conditioned by energy of receding contact lines, Applied Physics Letters 106 (2015) 241601.

[41] Y. Q. Zu, Y. Y. Yan, Single droplet on micro square post patterned surfaces - theoretical model and numerical simulation, Scientific Reports 6 (2016).

[42] T.M. Bucher, H.V. Tafreshi, Modeling air-water interface in disordered fibrous media with heterogeneous wettabilities, Colloids and Surfaces A: Physicochem. Eng. Aspects 461 (2014) 323-335.

[43] B. Zhang, J. Wang, Z. Liu, X. Zhang, Beyond Cassie equation: Local structure of heterogeneous surfaces determines the contact angles of microdroplets, Sci. Rep. 4 (2014) 5822.

[44] A. Marmur, From hydrophilic to superhydrophobic: Theoretical conditions for making high-contact-angle surfaces from low-contact-angle materials, Langmuir 24 (2008) 7573-7579. 


\section{FIGURE CAPTIONS}

Figure 1: (a) An example SEM image of an electrospun superhydrophobic Polystyrene coating with two layers of orthogonal fibers. (b) Apparent CA measured using a $0.5 \mu \mathrm{l}$ water droplet for coatings with different solid area fractions. Average fiber diameter was measured to be $5 \mu \mathrm{m}$.

Figure 2: Schematic representation of our virtual coatings made of two layers of parallel $(a-b)$ or orthogonal fibers (c-d).

Figure 3: Balance of forces across the AWI for parallel layers of fibers in (a), for orthogonal layers of fibers viewed from a direction along the fibers in the top layer in (b), and for orthogonal layers of fibers viewed from a direction perpendicular to the fibers in the top layer in (c).

Figure 4: (a) Schematic representation of AWI coming into contact with the substrate for a coating with two parallel layers of fibers. (b) Transition pressure versus fiber spacing in coatings with parallel fiber having different fiber diameter in the top and bottom layers. $\theta_{1}^{Y L}=\theta_{2}^{Y L}=100^{\circ}, d_{1}^{f}=5 \mu \mathrm{m}$.

Figure 5: Schematic representation of AWI coming into contact with the substrate for a coating with two orthogonal layers of fibers, with the view along the fibers of the top layer in (a) and along the fibers in the bottom layer in (b). Transition pressure versus fiber spacing in coatings with orthogonal fibers having different fiber diameter in the top and bottom layers in given in (c) for $\theta_{1}^{Y L}=\theta_{2}^{Y L}=100^{\circ}, d_{1}^{f}=5 \mu \mathrm{m}$. Transition pressures obtained for orthogonal and parallelfiber coatings are compared with one another in (d) for $d^{f}=10 \mu \mathrm{m}$.

Figure 6: Schematic representation of departure from the Cassie state due to AWI coalescing is given in (a) for coatings with parallel layers of fibers and in (b) and (c) for coatings with orthogonal fibers. Figures (b) and (c) are views along the fibers in the top layer and along the fibers in the bottom layer, respectively.

Figure 7: Transition pressure versus $s_{2}$ for coatings with orthogonal and parallel layers of fiber is given in (a) for $d_{1}^{f}=1 \mu \mathrm{m}, d_{2}^{f}=20 \mu \mathrm{m}, \theta_{1}^{Y L}=\theta_{2}^{Y L}=100^{\circ}, s_{1}=30 \mu \mathrm{m}$ for coatings with orthogonal fibers, and $s_{1}=s_{2}$ for coatings with parallel fibers. Droplet AWI coming into contact with the substrate on coatings with parallel and orthogonal layers of fiber are shown in (b) and (c), respectively. Here $V^{d}=0.524 \mu 1, \theta^{Y L}=100^{\circ}, d^{f}=10 \mu \mathrm{m}, s=170 \mu \mathrm{m}$.

Figure 8: Critical fiber spacing versus fiber diameter for coatings with parallel and orthogonal layers of fiber.

Figure 9: Example simulation results showing a droplet on coatings with parallel fibers from longitudinal and transverse views: (a) $d^{f}=5 \mu \mathrm{m}$, (b) $d^{f}=15 \mu \mathrm{m}$, and (c) $d^{f}=25 \mu \mathrm{m}$ with $\theta^{Y L}=100^{\circ}$. Apparent CA and coatings wetted area in the 
longitudinal and transvers directions are shown for coatings with different fiber diameters with $\theta^{Y L}=85^{\circ}$ and $\theta^{Y L}=100^{\circ}$ in (d) and (e), respectively. The inset figure in (e) shows the wetted length under the droplet.

Figure 10: Bottom views of a droplet with a volume of $0.524 \mu 1$ deposited on coatings with parallel or orthogonal layers of fiber with different fiber spacing having $\theta^{Y L}=100^{\circ}$ and $d^{f}=10 \mu \mathrm{m}$. For (a) through (c) and the droplet is in contact with the top layer only ( $s=40 \mu \mathrm{m}, s=85 \mu \mathrm{m}$, and $s=105 \mu \mathrm{m}$ in (a), (b), and (c), respectively). For (d) through (f) the droplet is in contact with both layers of a coating with parallel fibers ( $s=120 \mu \mathrm{m}, s=150 \mu \mathrm{m}$, and $s=160 \mu \mathrm{m}$ in (d), (e), and (f), respectively). For (g) through (i) the droplet is in contact with both layers of a coating with orthogonal fibers ( $s=120 \mu \mathrm{m}, s=170 \mu \mathrm{m}$, and $s=190 \mu \mathrm{m}$ in $(\mathrm{g})$, (h), and (i), respectively). Longitudinal and transvers CAs versus fiber spacing for $\theta^{Y L}=85^{\circ}$ and $\theta^{Y L}=100^{\circ}$ are given in (j) and (k), respectively. 

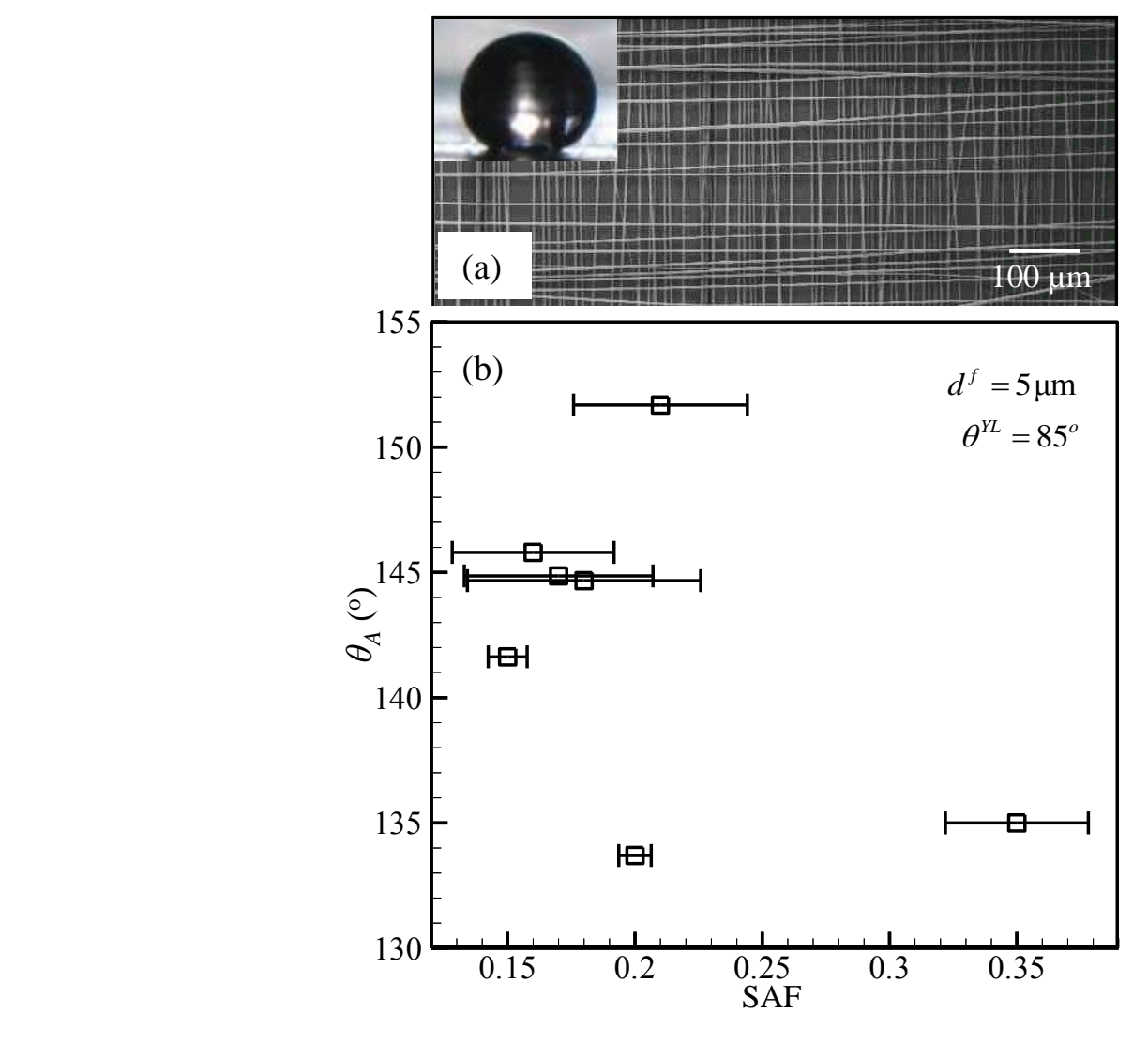

Fig. 1:

Figure(s)

-

(a)

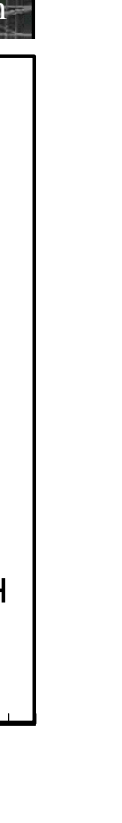
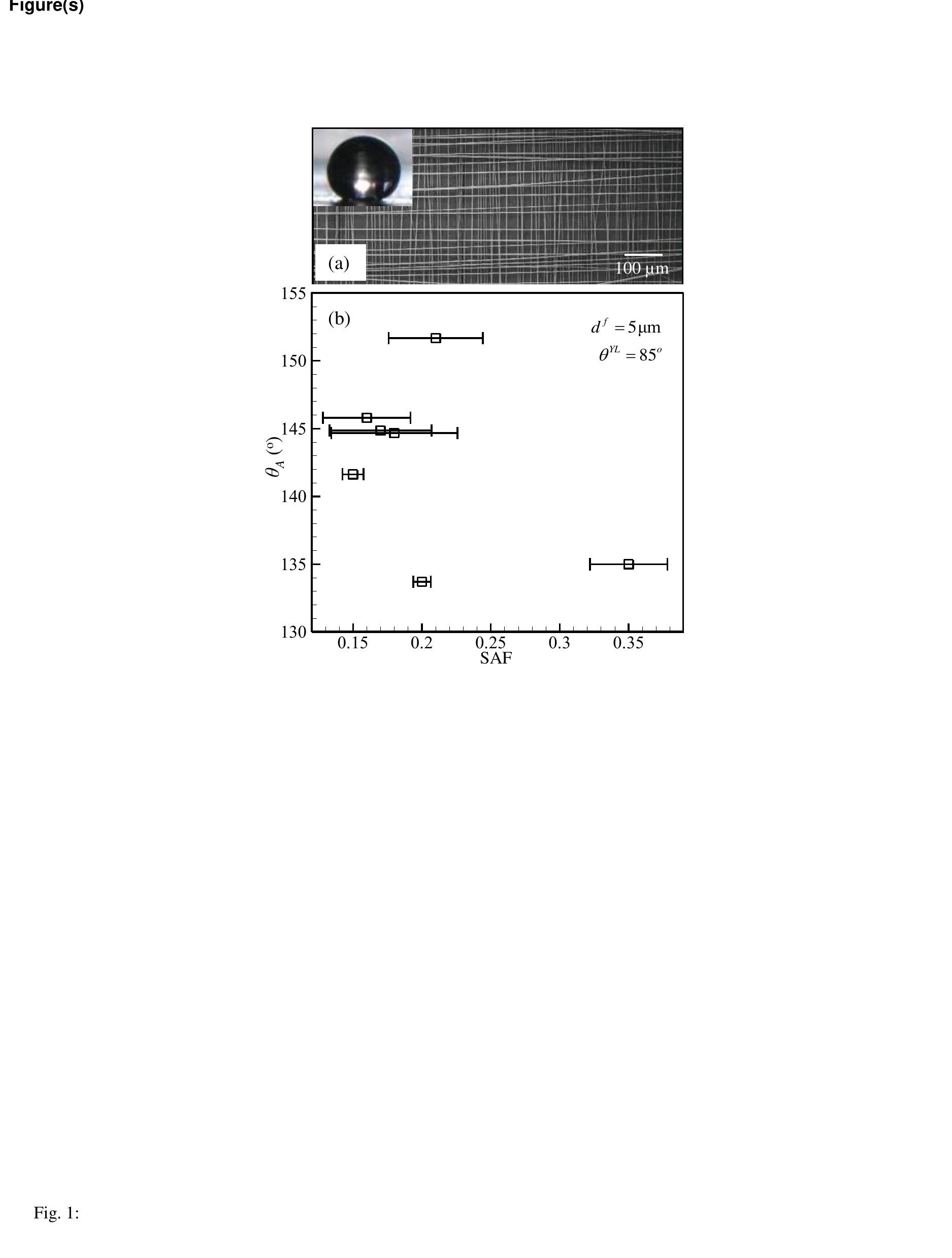
列 


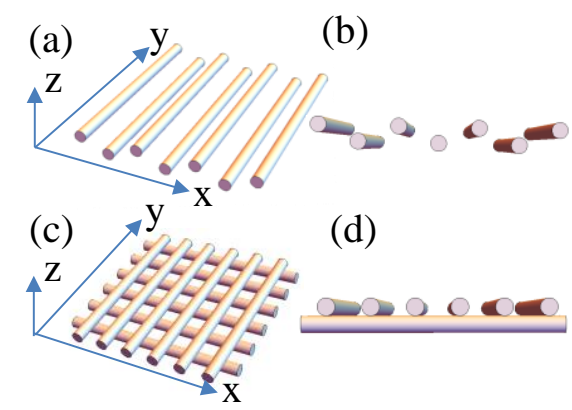

Fig. 2: 

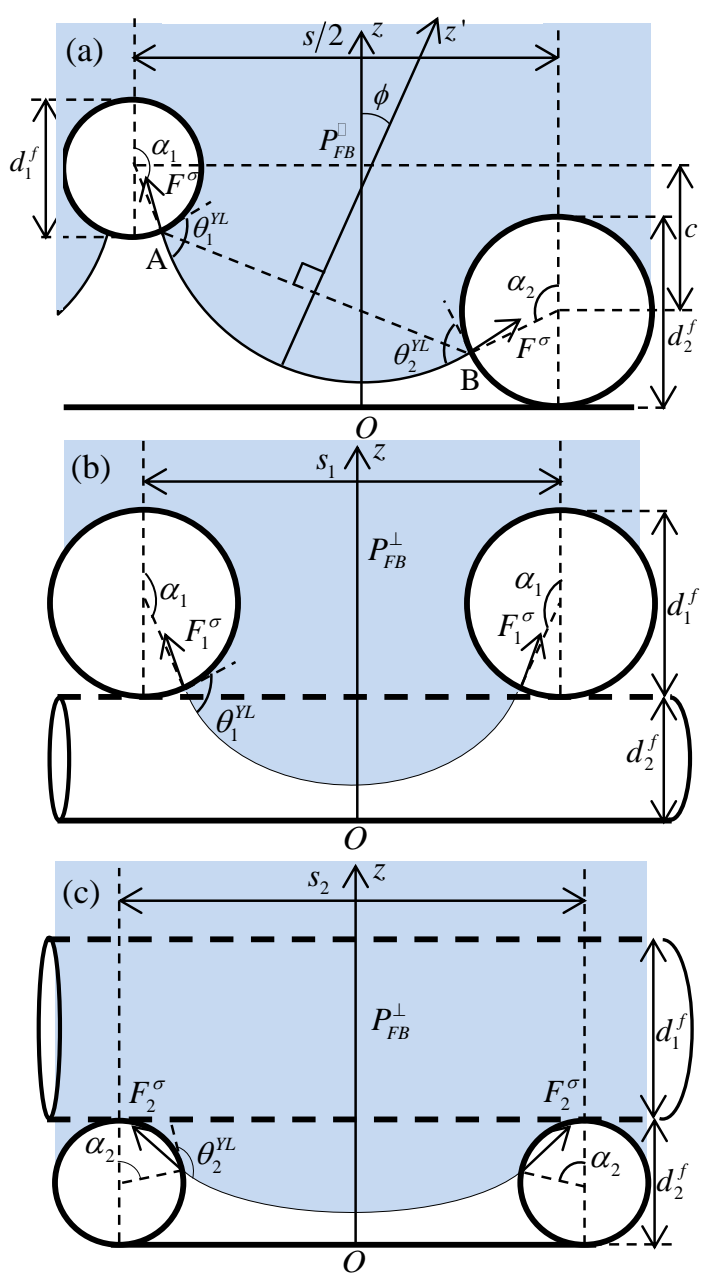

Fig. 3: 


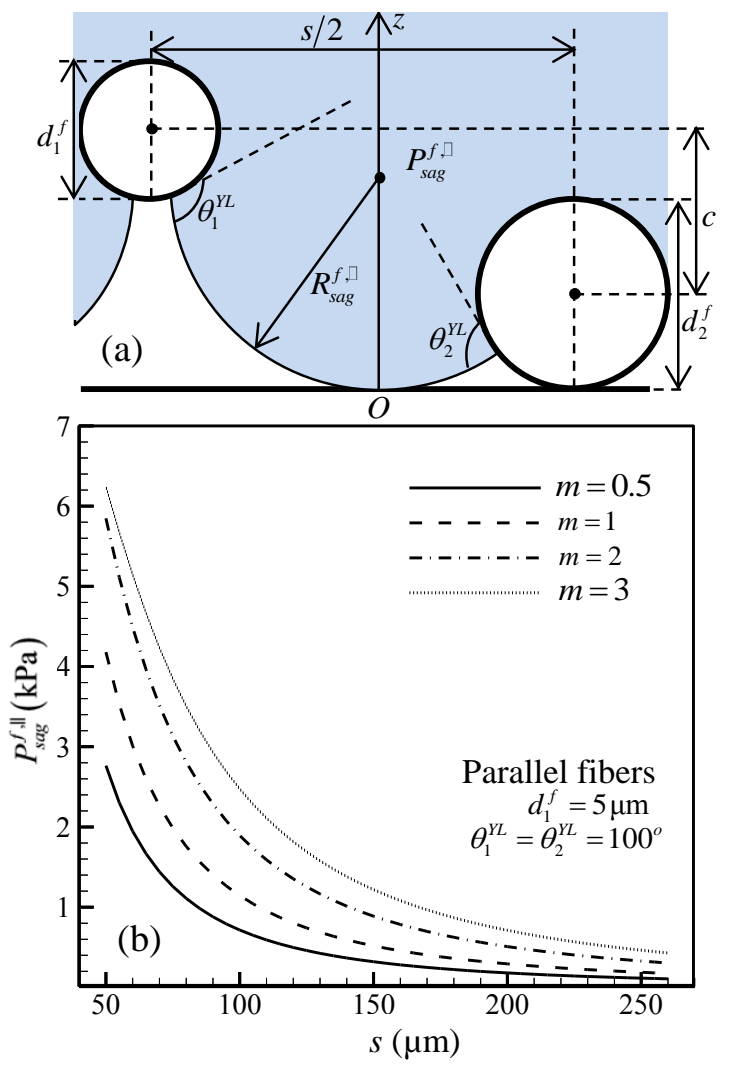

Fig. 4: 

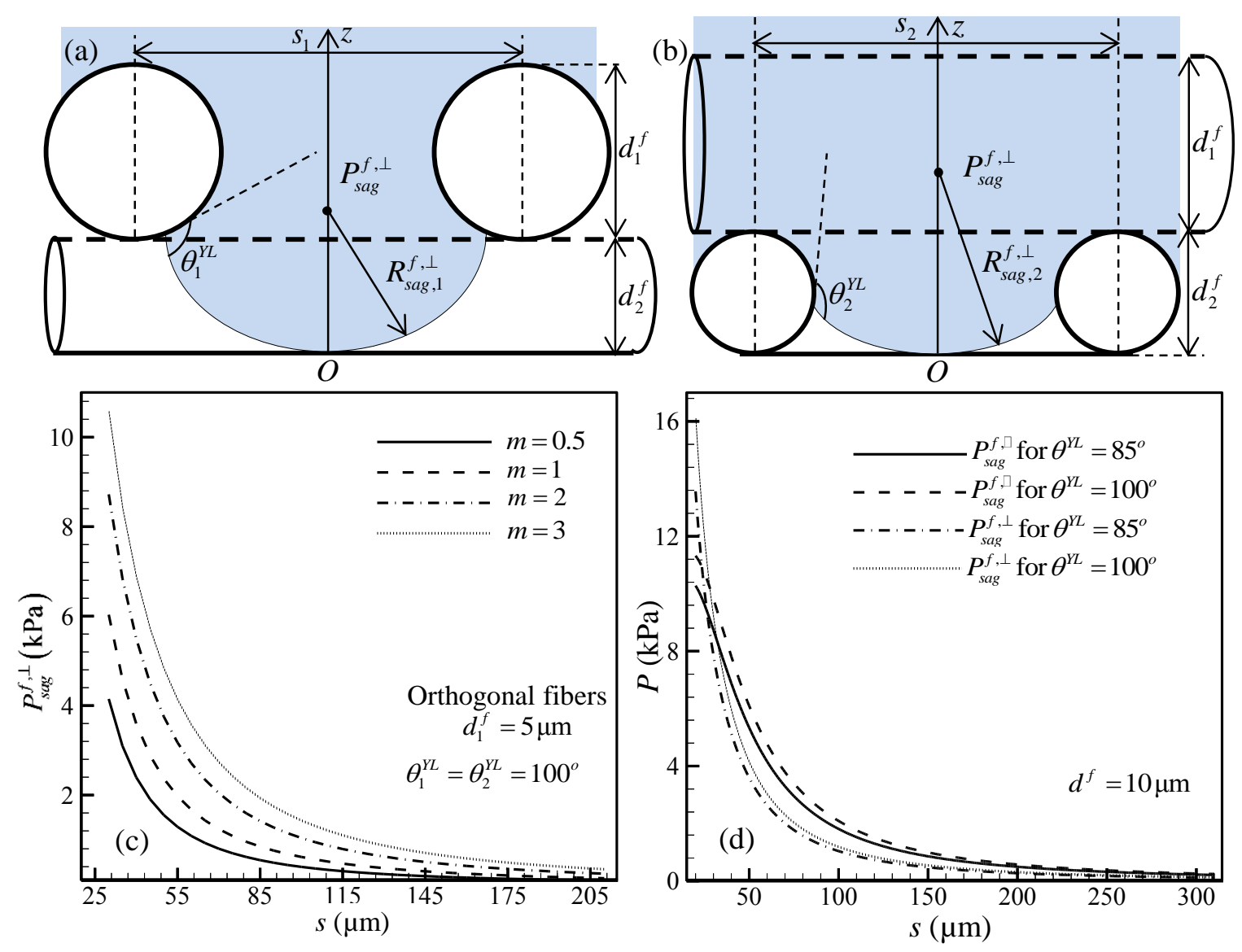

Fig. 5: 


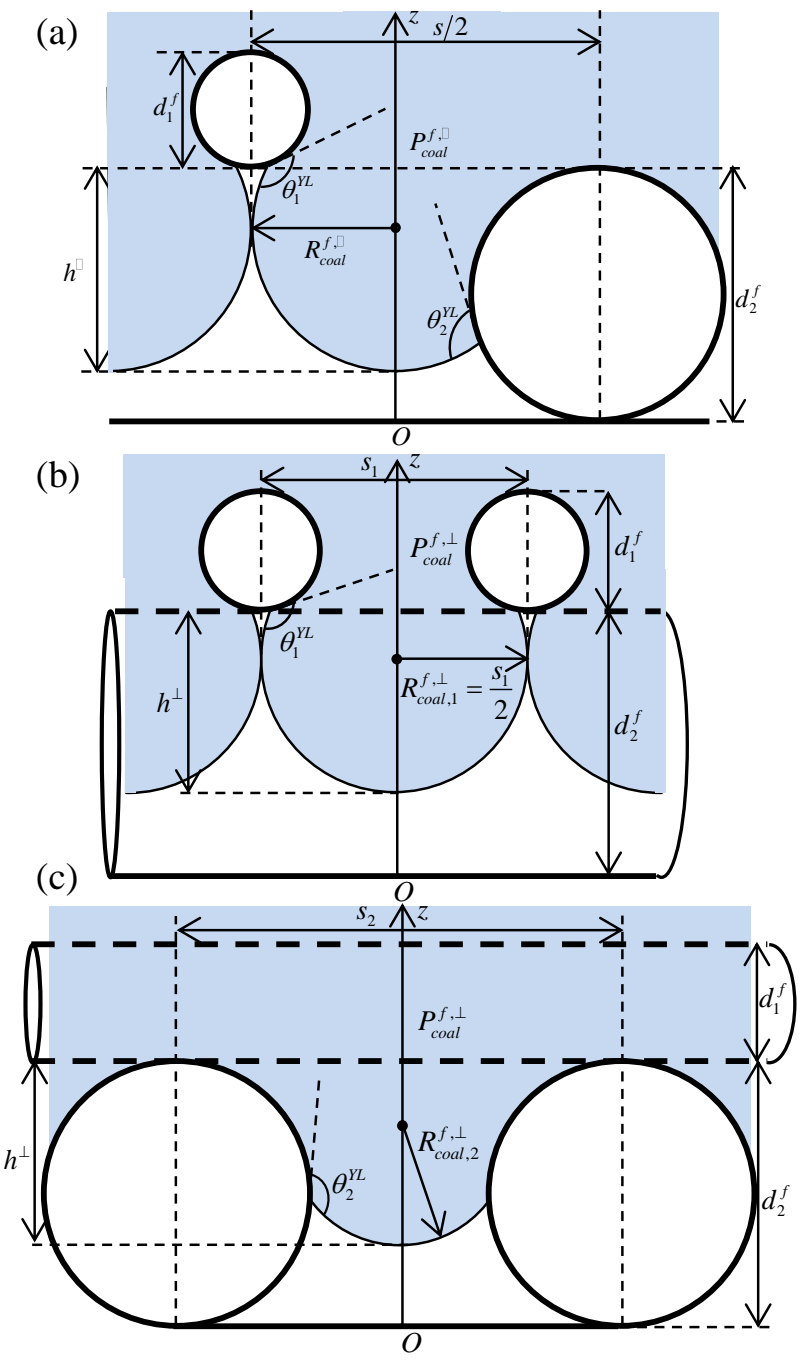

Fig. 6: 


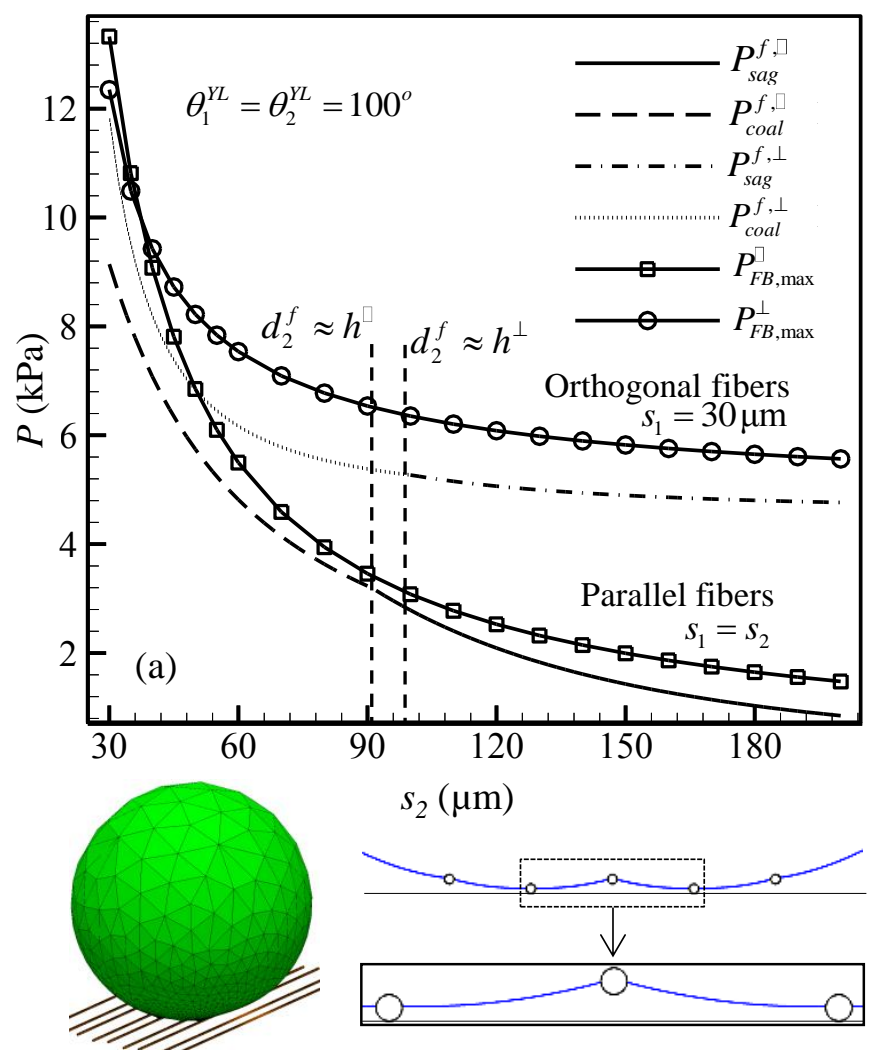

(b) $d^{f}=10 \mu \mathrm{m}, s=170 \mu \mathrm{m}$, and $\theta^{Y L}=100^{\circ}, V^{d}=0.524 \mathrm{~mm}^{3}$
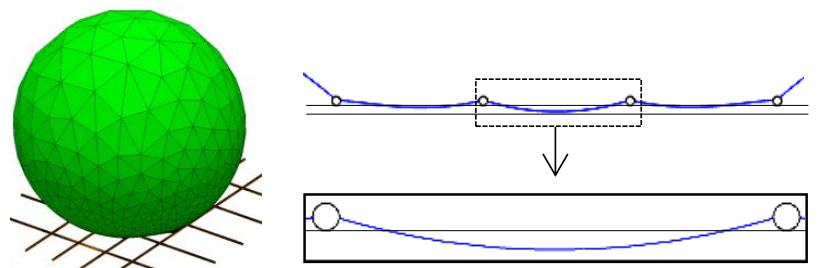

(c) $d^{f}=10 \mu \mathrm{m}, s=170 \mu \mathrm{m}$, and $\theta^{Y L}=100^{\circ}, V^{d}=0.524 \mathrm{~mm}^{3}$

Fig. 7: 


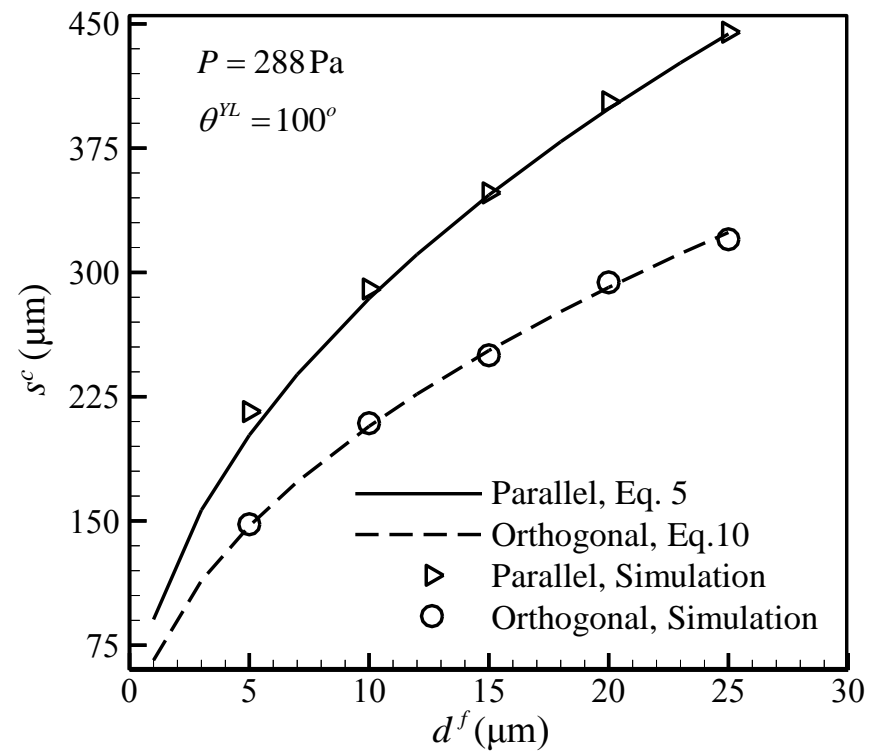

Fig. 8: 


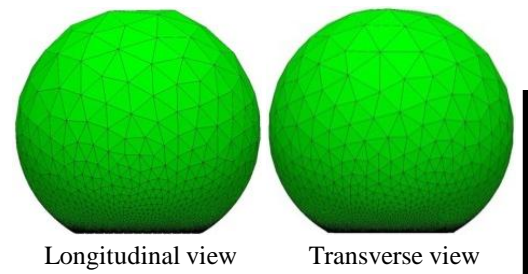

(a) $d^{f}=5 \mu \mathrm{m}$

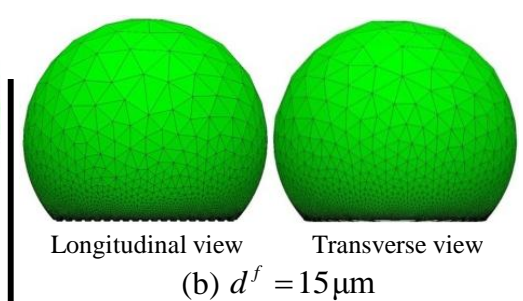

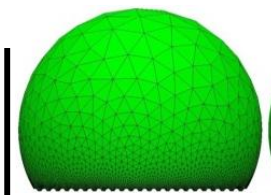

Longitudinal view

(c) $d^{f}=25 \mu \mathrm{m}$
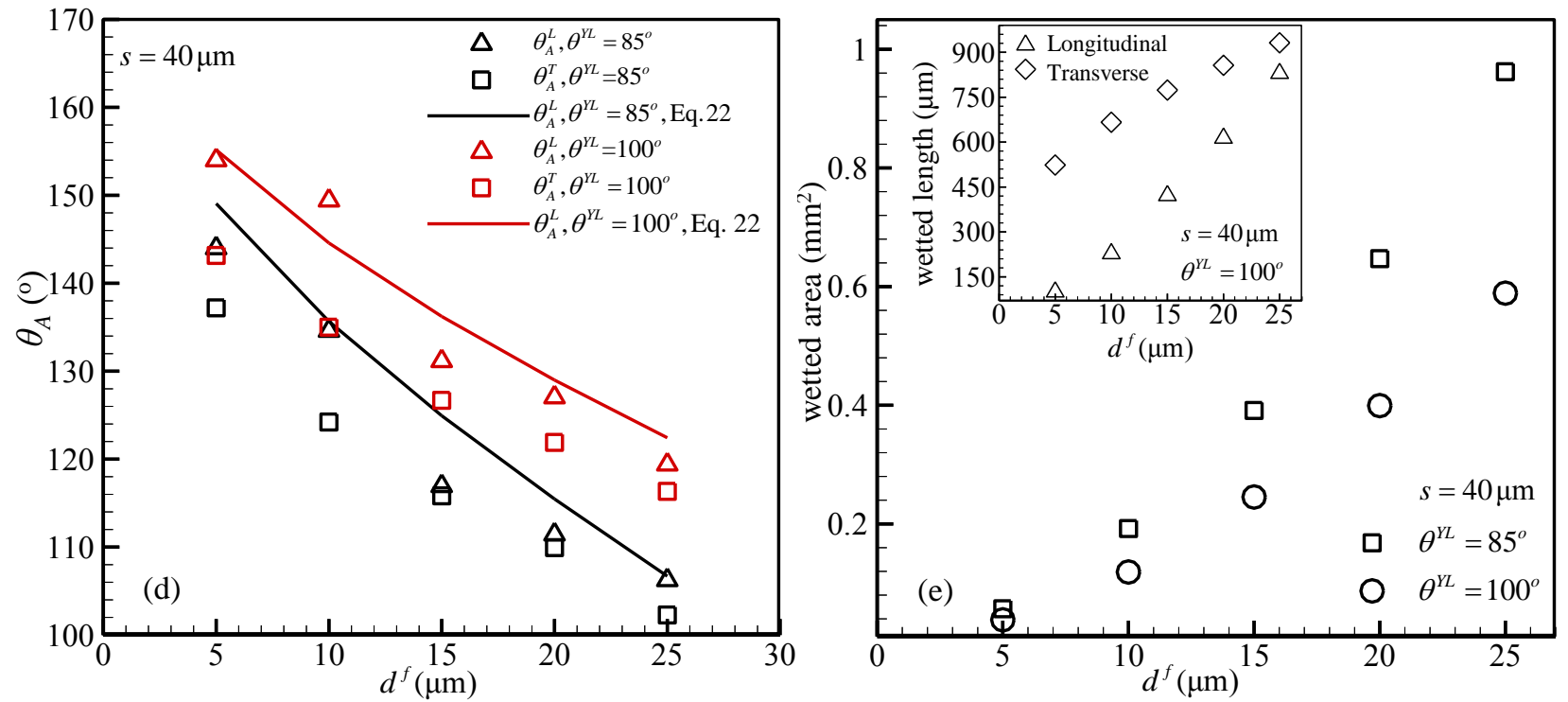

Fig. 9: 


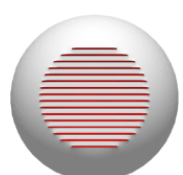

(a)

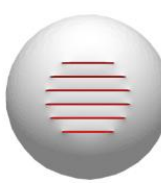

(b)

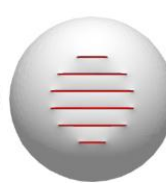

(c)

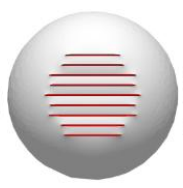

(d)

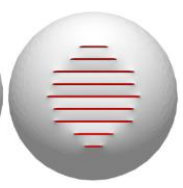

(e)

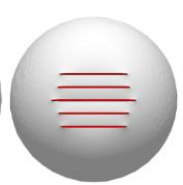

(f)

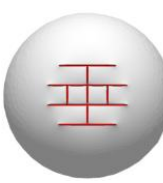

(g)

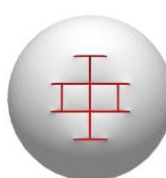

(h)

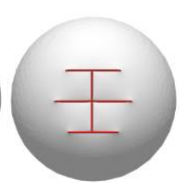

(i)
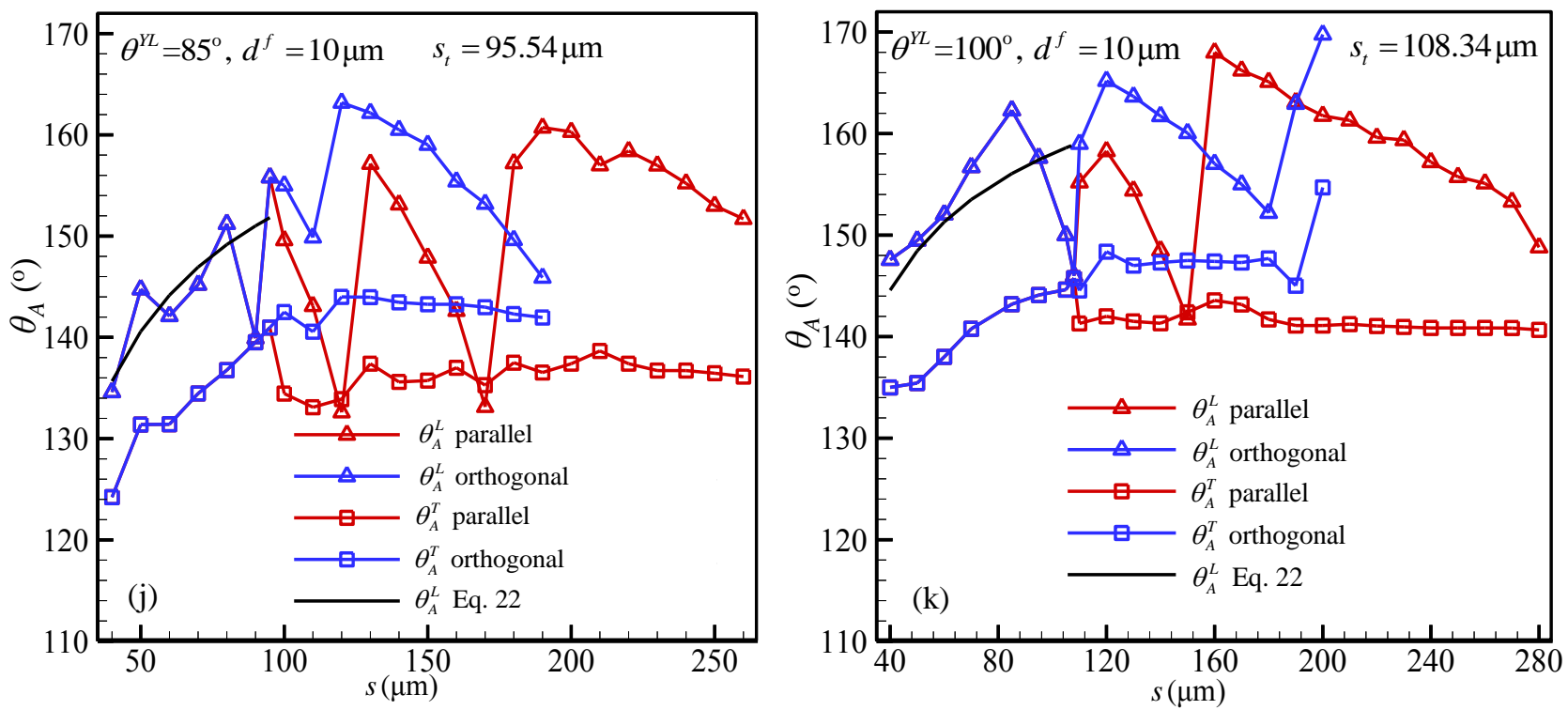

Fig. 10: 


\section{Graphical Abstract}
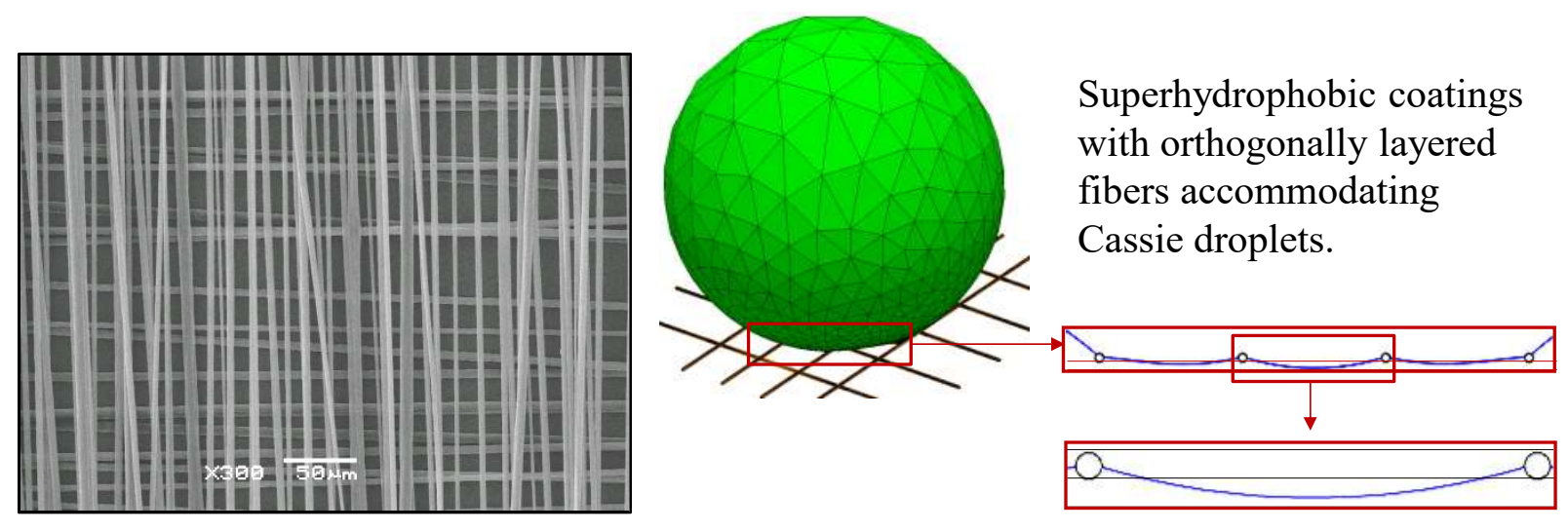